PROCEEDINGS OF THE

AMERICAN MATHEMATICAL SOCIETY

Volume 130, Number 6, Pages 1645-1654

S 0002-9939(02)05997-X

Article electronically published on January 25, 2002

\title{
ON THE DUAL OF ORLICZ-LORENTZ SPACE
}

\author{
H. HUDZIK, A. KAMIŃSKA, AND M. MASTYŁO
}

(Communicated by Dale Alspach)

\begin{abstract}
A description of the Köthe dual of the Orlicz-Lorentz space $\Lambda_{\varphi, w}$ generated by an Orlicz function $\varphi$ and a regular weight function $w$ is presented. It is also shown that in the case of separable Orlicz-Lorentz spaces the regularity condition on $w$ is necessary and sufficient for the coincidence of the Banach dual space with the described Köthe dual space.
\end{abstract}

\section{INTRODUCTION}

Let $(\Omega, \mu):=(\Omega, \Sigma, \mu)$ be a measure space with the complete and $\sigma$-finite measure $\mu$, and let $L^{0}(\mu)$ denote the space of all $\mu$-equivalence classes of $\Sigma$-measurable functions on $\Omega$ with the topology of convergence in measure on $\mu$-finite sets.

A Banach space $\left(E,\|\cdot\|_{E}\right)$ is said to be a Banach function space on $(\Omega, \mu)$ if it is a subspace of $L^{0}(\mu)$ such that there exists $h \in L^{0}(\mu)$ with $h>0$ a.e. in $\Omega$ and the assumptions that $f \in L^{0}(\mu), g \in E$ and $|f| \leq|g|$ a.e. in $\Omega$ imply $f \in E$ and $\|f\|_{E} \leq\|g\|_{E}$. If in addition the unit ball $B_{E}=\left\{f:\|f\|_{E} \leq 1\right\}$ is closed in $L^{0}(\mu)$, then we say that $E$ has the Fatou property. A Banach function space defined on $\left(\mathbb{N}, 2^{\mathbb{N}}, \mu\right)$ with the counting measure $\mu$ is called a Banach sequence space (on $\mathbb{N}$ ).

A Banach function space $E$ on $(\Omega, \mu)$ is said to be symmetric if for every $f \in$ $L^{0}(\mu)$ and $g \in E$ with $\mu_{f}=\mu_{g}$, we have $f \in E$ and $\|f\|_{E}=\|g\|_{E}$, where for any $h \in L^{0}(\mu), \mu_{h}$ is the distribution function defined by

$$
\mu_{h}(t)=\mu(\{\omega \in \Omega:|h(\omega)|>t\}), t \geq 0 .
$$

If $E$ is a Banach function space on $(\Omega, \mu)$, then the Köthe dual $E^{\prime}$ of $E$ is a Banach function space, which can be identified with the space of all functionals possessing an integral representation, that is,

$$
E^{\prime}=\left\{g \in L^{0}(\mu):\|g\|_{E^{\prime}}=\sup _{\|f\|_{E} \leq 1} \int_{\Omega}|f g| d \mu<\infty\right\} .
$$

It is well known that if $E$ has order continuous norm (i.e., $\left\|f_{n}\right\|_{E} \rightarrow 0$ whenever $\left.E \ni f_{n} \downarrow 0\right)$, then the dual space $E^{*}$ can be naturally identified with $E^{\prime}([]$ ).

In this paper we are interested in the description of the Köthe duals for symmetric Orlicz-Lorentz spaces defined on either nonatomic or purely atomic measure space. Since after minor modifications the proofs presented in the paper work in essentially

Received by the editors September 24, 1999 and, in revised form, April 20, 2000.

1991 Mathematics Subject Classification. Primary 46B10, 46 E30.

The research of the second and third authors was supported by NATO Collaborative Grant CRG 972918. 
the same way in both cases, for simplicity, we consider only the case of OrliczLorentz spaces defined on $(I, m)$, where either $I=(0,1)$ or $I=(0, \infty)$ and $m$ is the Lebesgue measure.

We recall that if $\varphi:[0, \infty) \rightarrow[0, \infty)$ is an Orlicz function (i.e., a convex function which assumes value zero only at zero) and $w: I \rightarrow(0, \infty)$ is a weight function (i.e., nonincreasing and locally integrable with respect to the measure $m$ and such that $\int_{0}^{\infty} w d m=\infty$ if $\left.I=(0, \infty)\right)$, then the Orlicz-Lorentz function space $\Lambda_{\varphi, w}$ on $(\Omega, \mu)$ is the set of all $f \in L^{0}(\mu)$ such that

$$
\int_{\Omega} \varphi\left(\lambda f^{*}\right) w d m<\infty
$$

for some $\lambda>0$, where for any $f \in L^{0}(\mu), f^{*}$ denotes the nonincreasing rearrangement of $f$ defined by

$$
f^{*}(t)=\inf \left\{\lambda>0: \mu_{f}(\lambda) \leq t\right\}
$$

for any $t>0$ (by convention $\inf \emptyset=\infty$ ).

In the case of counting measure on $2^{\mathbb{N}}$ the Orlicz-Lorentz sequence space $\lambda_{\varphi, w}$ on $\mathbb{N}$ is defined by

$$
\lambda_{\varphi, w}=\left\{x=\{x(k)\}: \sum_{k=1}^{\infty} \varphi\left(\lambda x^{*}(k)\right) w(k)<\infty \text { for some } \lambda>0\right\} .
$$

Here $w=\{w(k)\}$ is a weight sequence, a nonincreasing sequence of positive reals such that $\sum_{k=1}^{\infty} w(k)=\infty$.

It is easy to check that $\Lambda_{\varphi, w}$ (resp., $\lambda_{\varphi, w}$ ) is a symmetric function space (resp., symmetric sequence space) with the Fatou property, equipped with the norm

$$
\|f\|=\inf \left\{\lambda>0: \int_{I} \varphi\left(f^{*} / \lambda\right) w d m \leq 1\right\}
$$

respectively

$$
\|x\|=\inf \left\{\lambda>0: \sum_{k=1}^{\infty} \varphi\left(x^{*}(k) / \lambda\right) w(k) \leq 1\right\} .
$$

Note that if $w \equiv 1$ (resp., $w(k)=1$ for all $k \in \mathbb{N}$ ), then $\Lambda_{\varphi, w}$ (resp., $\lambda_{\varphi, w}$ ) is the Orlicz function space $L_{\varphi}$ (resp., Orlicz sequence space $\ell_{\varphi}$ ). If $\varphi(t)=t$, then $\Lambda_{\varphi, w}$ (resp., $\lambda_{\varphi, w}$ ) is the Lorentz space $\Lambda_{w}$ (resp., $\lambda_{w}$ ). We recall that an Orlicz function $\varphi$ satisfies the $\Delta_{2}$-condition $\left(\varphi \in \Delta_{2}\right)$ if there exists $C>0$ such that $\varphi(2 t) \leq C \varphi(t)$ for all $t>0$. We will further say that $\varphi$ is an $N$-function whenever $\lim _{t \rightarrow 0} \varphi(t) / t=0$ and $\lim _{t \rightarrow \infty} \varphi(t) / t=\infty$. We refer to [5] and 7] to study the basic properties of Orlicz-Lorentz spaces as well to the references included therein.

In what follows by a regular weight we mean a weight function $w$ such that, if we denote $S(t)=\int_{0}^{t} w(s) d s$ for $t \in I$, then $S(2 t) \geq K S(t)$ for any $t>0(t \in(0,1 / 2)$ in the case $I=(0,1))$, where $K>1$ is independent of $t$. In the sequence case a weight $w=\{w(k)\}$ is regular if $S(2 n) \geq K S(n)$ for any $n \in \mathbb{N}$, where $S(n)=\sum_{k=1}^{n} w(k)$ and $K>1$ is a constant independent of $n$. It is well known and easy to show that $w$ is regular iff there exists $C>0$ such that $t w(t) \leq S(t) \leq C t w(t)$ for all $t \in I$ in the function case and analogously $n w(n) \leq S(n) \leq C n w(n)$ for all $n \in \mathbb{N}$ in the sequence case. 
Recall that if $\rho: I \rightarrow(0, \infty)$ is a concave function, then the Marcinkiewicz space $M_{\rho}$ is defined by

$$
M_{\rho}=\left\{f \in L^{0}(m):\|f\|_{M_{\rho}}=\sup _{t \in I} \frac{\int_{0}^{t} f^{*}(s) d s}{\rho(t)}<\infty\right\},
$$

and the Marcinkiewicz space $M_{S}$ with $S(t)=\int_{0}^{t} w(s) d s$ is the Köthe dual of $\Lambda_{w}$, that is,

$$
\left(\Lambda_{w}\right)^{\prime}=M_{S}
$$

with equality of norms (see [3] or [9]). In what follows we will write $f \asymp g$ for nonnegative functions $f$ and $g$ whenever $C_{1} f \leq g \leq C_{2} f$ for some $C_{j}>0, j=1,2$.

The problem of the description of dual spaces for Lorentz sequence spaces has been considered in [1] and [2]. It was proved there that the regularity of $w$ is a necessary and sufficient condition, in the case $p=1$, and a sufficient condition in the case $p>1$, in order that the Köthe dual of classical Lorentz sequence space $d(w, p)=\lambda_{\varphi, w}$ with $\varphi(t)=t^{p}$ for $t \geq 0$ to consist exactly of those sequences $\{x(k)\}$ for which $\left\{x^{*}(k) / w(k)^{1 / p}\right\} \in \ell_{p^{\prime}}$, where $1 / p+1 / p^{\prime}=1$.

In [1] Allen also raises the question of whether the similar description of the Köthe dual space is also true for Lorentz function spaces. Reisner [12] answered this question positively and gave a proof which works in essentially the same way in the sequence and in the function spaces. He also proved that regularity of $w$ is necessary for $p>1$.

Following the ideas from [12] we prove in this paper, under the assumption that $\varphi$ is an $N$-function satisfying the $\Delta_{2}$-condition, that the regularity of the weight function $w$ is a necessary and sufficient condition for the dual of the Orlicz-Lorentz space $\Lambda_{\varphi, w}$ on $(I, m)$ to consist exactly of those functions $f$ for which $f^{*} / w$ belongs to the Orlicz function space $L_{\varphi_{*}}$ on $(I, w d m)$, where $\varphi_{*}$ is the Young conjugate of $\varphi$, i.e.,

$$
\varphi_{*}(t)=\sup \{s t-\varphi(s): s \geq 0\}
$$

for $t \geq 0$. We also obtain some partial results for $\varphi$ being an arbitrary Orlicz function. A similar result is also true for the symmetric Orlicz-Lorentz sequence space $\lambda_{\varphi, w}$ on $\mathbb{N}$.

In the proof of the main result we will use the Lozanovskir theorem on the representation of the Köthe dual space for the Calderón-Lozanovskiu space (see [11]). Let us recall that if $\left(E_{0}, E_{1}\right)$ is any couple of Banach function spaces on $(\Omega, \mu)$ and $\mathcal{U}$ denotes the set of all concave and positively homogeneous functions $\psi:[0, \infty) \times[0, \infty) \rightarrow[0, \infty)$ such that $\psi(s, t)=0$ if and only if $s=t=0$, then the Calderón-Lozanovskiu space $\psi\left(E_{0}, E_{1}\right)$ generated by the couple $\left(E_{0}, E_{1}\right)$ and a function $\psi \in \mathcal{U}$ consists of all $f \in L^{0}(\mu)$ such that $|f| \leq \lambda \psi\left(\left|f_{0}\right|,\left|f_{1}\right|\right)$ a.e. for some $\lambda>0$ and $f_{j} \in E_{j}, j=0,1$. The space $\psi\left(E_{0}, E_{1}\right)$ is a Banach function space on $(\Omega, \mu)$ (cf. [10, [11]) equipped with the following equivalent norms:

$$
\|f\|_{\psi}=\inf \left\{\lambda>0:|f| \leq \lambda \psi\left(\left|f_{0}\right|,\left|f_{1}\right|\right) \text { a.e., }\left\|f_{j}\right\|_{E_{j}} \leq 1, j=0,1\right\}
$$

and

$$
\|f\|_{\psi}^{1}=\inf \left\{\left\|f_{0}\right\|_{E_{0}}+\left\|f_{1}\right\|_{E_{1}}:|f|=\psi\left(\left|f_{0}\right|,\left|f_{1}\right|\right)\right\},
$$

satisfying the inequalities

$$
\|f\|_{\psi} \leq\|f\|_{\psi}^{1} \leq 2\|f\|_{\psi}
$$


for all $f \in \psi\left(E_{0}, E_{1}\right)$. In the case of the power function $\psi_{\theta}(s, t)=s^{1-\theta} t^{\theta}$ with $0<\theta<1, \psi_{\theta}\left(E_{0}, E_{1}\right)$ is the well-known Calderón space $E_{0}^{1-\theta} E_{1}^{\theta}$ (see [4]).

For any $\psi \in \mathcal{U}$ the involution $\widehat{\psi}$ of $\psi$ is defined on $\mathbb{R}_{+}^{2}$ by

$$
\widehat{\psi}(s, t)=\inf \left\{\frac{\alpha s+\beta t}{\psi(\alpha, \beta)}: \alpha, \beta>0\right\} .
$$

Lozanovskiǔ [11] proved the following theorem (see also [10], [13]).

Theorem 1. a) If $E_{0}, E_{1}$ are two Banach function spaces on the same measure space, then for all $\psi \in \mathcal{U}$ we have

$$
\left(\psi\left(E_{0}, E_{1}\right),\|\cdot\|_{\psi}\right)^{\prime}=\left(\widehat{\psi}\left(E_{0}^{\prime}, E_{1}^{\prime}\right),\|\cdot\|_{\widehat{\psi}}^{1}\right)
$$

with equality of the norms.

b) For every $0 \leq f \in L^{1}(\mu)$ and $\varepsilon>0$, there exist $0 \leq g \in E, 0 \leq h \in E^{\prime}$ such that $f=g h$ and

$$
\|g\|_{E}\|h\|_{E^{\prime}} \leq(1+\varepsilon)\|f\|_{L^{1}}
$$

If $E$ has the Fatou property, we may take $\varepsilon=0$ in the above inequality.

\section{REsults}

The main aim of this section is to prove a representation theorem for the Köthe dual of Orlicz-Lorentz spaces. The proof for sequence spaces is analogous and more simple than in the function case. Thus we limit ourselves to a proof for function spaces only. We begin with the lemma which will be useful in the sequel.

Lemma 1. Let $\psi \in \mathcal{U}$ and let $f, g \in L^{0}(\mu)$ be such that $\mu_{f}(t)<\infty$ and $\mu_{g}(t)<\infty$ for every $t>0$. If $h \in L^{0}(\mu)$ is such that $|h| \leq \psi(|f|,|g|)$ a.e., then for all $t>0$

$$
h^{*}(t) \leq 2 \psi\left(f^{*}(t / 2), g^{*}(t / 2)\right) .
$$

Proof. Since each $\psi \in \mathcal{U}$ is nondecreasing in each variable, we have $\psi(s, t) \leq$ $\max \{s / \alpha, t / \beta\} \psi(\alpha, \beta)$, and in consequence

$$
\psi(s, t) \leq\left(\frac{s}{\alpha}+\frac{t}{\beta}\right) \psi(\alpha, \beta)
$$

for all $s, t \geq 0$ and $\alpha, \beta>0$. This implies that

$$
\psi(s, t) \leq \tilde{\psi}(s, t) \leq 2 \psi(s, t)
$$

holds for all $s, t \geq 0$, where

$$
\tilde{\psi}(s, t):=\inf _{\alpha, \beta>0}(s / \alpha+t / \beta) \psi(\alpha, \beta)
$$

for all $s, t \geq 0$. Now fix $\alpha, \beta>0$. Then applying the above inequalities, we conclude that for all $\alpha, \beta>0$

$$
|h| \leq\left(\frac{|f|}{\alpha}+\frac{|g|}{\beta}\right) \psi(\alpha, \beta) \text { a.e. }
$$

and in view of the inequality $(f+g)^{*}(t) \leq f^{*}(t / 2)+g^{*}(t / 2)$,

$$
h^{*}(t) \leq\left(\frac{f^{*}(t / 2)}{\alpha}+\frac{g^{*}(t / 2)}{\beta}\right) \psi(\alpha, \beta)
$$


for all $t>0$. Hence

$$
h^{*}(t) \leq \tilde{\psi}\left(f^{*}(t / 2), g^{*}(t / 2)\right) \leq 2 \psi\left(f^{*}(t / 2), g^{*}(t / 2)\right)
$$

for all $t>0$, which completes the proof.

In what follows, given an Orlicz function $\varphi$, we define $I(f)=\int_{I} \varphi_{*}\left(f^{*} / w\right) w d m$ for $f \in L^{0}(m)$ and

$$
M_{\varphi_{*}, w}=\left\{f \in L^{0}(m): I(f / \lambda)<\infty \text { for some } \lambda>0\right\} .
$$

In the space $M_{\varphi_{*}, w}$ we define an order monotone and homogenous functional

$$
\|f\|_{M_{\varphi_{*}, w}}=\inf \{\lambda>0: I(f / \lambda) \leq 1\} .
$$

One can show that if $w$ is regular, then the functional $\|\cdot\|_{M_{\varphi_{*}, w}}$ is a quasinorm. We also observe that if $\varphi(t)=t$, then

$$
M_{\varphi_{*}, w}=\left\{f \in L^{0}(m):\|f\|_{M_{\varphi_{*}, w}}=\sup _{t \in I} \frac{f^{*}(t)}{w(t)}<\infty\right\}
$$

and $M_{\varphi_{*}, w}=M_{S}$ with $\|\cdot\|_{M_{\varphi_{*}, w}} \asymp\|\cdot\|_{M_{S}}$, by $t w(t) \asymp S(t)$, whenever $w$ is regular. In a similar way we define the sequence space $m_{\varphi_{*}, w}$ for a sequence weight $w$.

Before we prove the main theorem we will need the following lemma which, in the case of $\varphi(t)=t^{p}$ with $1 \leq p<\infty$, has been proved in [12] as Lemma 2 with means of much more involved arguments.

Lemma 2. Let an Orlicz function $\varphi$ and a weight funtion $w$ be such that $\left(\Lambda_{\varphi, w}\right)^{\prime}=$ $M_{\varphi_{*}, w}$. Then there is $K>0$ so that

$$
\|g\|_{M_{\varphi_{*}, w}} \leq K\|g\|_{\left(\Lambda_{\varphi, w}\right)^{\prime}} \text { for all } g \in\left(\Lambda_{\varphi, w}\right)^{\prime} .
$$

Proof. For a contradiction assume that there exists a sequence $\left\{g_{k}\right\}$ with

$$
\left\|g_{k}\right\|_{\left(\Lambda_{\varphi, w}\right)^{\prime}}=1 \text { and }\left\|g_{k}\right\|_{M_{\varphi_{*}, w}}>k 2^{k} \text { for all } k \in \mathbb{N} .
$$

Obviously for the function $g$ defined by

$$
g=\sum_{k=1}^{\infty} \frac{1}{2^{k}}\left|g_{k}\right|,
$$

we have $g \in\left(\Lambda_{\varphi, w}\right)^{\prime}=M_{\varphi_{*}, w}$. Since $g \geq\left|g_{k}\right| / 2^{k}$, we have $g^{*}(t) \geq g_{k}^{*}(t) / 2^{k}$ for all $k \in \mathbb{N}$ and $t \in I$. Thus from the order monotonicity and homogeneity of the functional $\|\cdot\|_{M_{\varphi_{*}, w}}$, we get

$$
\|g\|_{M_{\varphi_{*}, w}} \geq k
$$

for all $k \in \mathbb{N}$. This contradiction finishes the proof of the lemma.

Now we are ready to prove the main result of the paper.

Theorem 2. Let $w$ be a weight function and let either $\varphi(t)=t$ or $\varphi$ be an $N$-function. Then the following holds true:

(i) If $w$ is a regular weight, then $\left(\Lambda_{\varphi, w}\right)^{\prime}=M_{\varphi_{*}, w}$ and $\|\cdot\|_{\left(\Lambda_{\varphi, w}\right)^{\prime}} \asymp\|\cdot\|_{M_{\varphi_{*}, w}}$.

(ii) If $\varphi$ satisfies the $\Delta_{2}$-condition and $\left(\Lambda_{\varphi, w}\right)^{\prime}=M_{\varphi_{*}, w}$, then $w$ is regular. 
Proof. At first we notice that if $E$ is any Banach function space on a measure space $(\Omega, \mu), \varphi: \mathbb{R}_{+} \rightarrow \mathbb{R}_{+}$is an Orlicz function and $\psi \in \mathcal{U}$ is defined by $\psi(s, t)=0$ if $t=0$ and $\psi(s, t)=t \varphi^{-1}(s / t)$ if $t>0$, then

$$
\psi\left(E, L^{\infty}\right)=\left\{f \in L^{0}: \varphi \circ(\lambda f) \in E \text { for some } \lambda>0\right\}
$$

and

$$
\|f\|_{\psi}=\inf \left\{\lambda>0:\|\varphi \circ(f / \lambda)\|_{E} \leq 1\right\} .
$$

In particular, we obtain $\Lambda_{\varphi, w}=\psi\left(\Lambda_{w}, L^{\infty}\right)$. Hence by Theorem 1, we get

$$
\left(\Lambda_{\varphi, w}\right)^{\prime}=\widehat{\psi}\left(\left(\Lambda_{w}\right)^{\prime},\left(L^{\infty}\right)^{\prime}\right)^{\prime}=\widehat{\psi}\left(M_{S}, L^{1}\right),
$$

with equality of the norms, where $\widehat{\psi}\left(M_{S}, L^{1}\right)$ is considered with the norm $\|\cdot\|_{\widehat{\psi}}^{1}$. It is also easy to check that $\widehat{\psi}(s, t)=0$ for $s=0$ and $\widehat{\psi}(s, t)=s \varphi_{*}^{-1}(t / s)$ for $s>0$. Notice by the assumption that $\varphi$ is an $N$-function, $\varphi_{*}$ is an Orlicz function and thus $\widehat{\psi} \in \mathcal{U}$.

Now we are ready to prove (i). If $\varphi(t)=t$, then (i) is obvious by the observation stated before Lemma 2. Assume now that $\varphi$ is an $N$-function and let $f \in \widehat{\psi}\left(M_{S}, L^{1}\right)$ with $\|f\|_{\widehat{\psi}}^{1} \leq 1$. Since $\|f\|_{\widehat{\psi}} \leq\|f\|_{\widehat{\psi}}^{1}$, we get $\|f\|_{\widehat{\psi}} \leq 1$. This yields

$$
|f| \leq \widehat{\psi}\left(\left|f_{0}\right|,\left|f_{1}\right|\right) \text { a.e. }
$$

for some $f_{0} \in M_{S}$ and $f_{1} \in L^{1}$ with $\left\|f_{0}\right\|_{M_{S}} \leq 1$ and $\left\|f_{1}\right\|_{L^{1}} \leq 1$. In consequence, it follows by Lemma 1 that

$$
f^{*}(t) \leq 2 \widehat{\psi}\left(f_{0}^{*}(t / 2), f_{1}^{*}(t / 2)\right)
$$

for all $t \in I$. By regularity of $w$ for any $t \in I$,

$$
f_{0}^{*}(t / 2) / C w(t) \leq t f_{0}^{*}(t / 2) / S(t) \leq\left\|f_{0}^{*}(\cdot / 2)\right\|_{M_{S}} \leq 2\left\|f_{0}\right\|_{M_{S}} \leq 2
$$

and hence for $t \in I$,

$$
f_{0}^{*}(t / 2) \leq 2 C w(t)
$$

Combining the above inequalities, we obtain

$$
f^{*}(t) \leq 2 \widehat{\psi}\left(2 C w(t), f_{1}^{*}(t / 2)\right)
$$

for all $t \in I$. Therefore

$$
f^{*}(t) \leq 4 C w(t) \varphi_{*}^{-1}\left(f_{1}^{*}(t / 2) / 2 C w(t)\right)
$$

or equivalently

$$
\varphi_{*}\left(\frac{f^{*}(t)}{4 C w(t)}\right) w(t) \leq \frac{1}{2 C} f_{1}^{*}(t / 2)
$$

for any $t \in I$. Since $\left\|f_{1}\right\|_{L^{1}} \leq 1,\left\|f_{1}^{*}(\cdot / 2)\right\|_{L^{1}} \leq 2$, we obtain that $f^{*} \in M_{\varphi_{*}, w}$ and $\|f\|_{M_{\varphi_{*}, w}} \leq 4 C$. In consequence we proved that for any $f \in\left(\Lambda_{\varphi, w}\right)^{\prime}$, we have

$$
\|f\|_{M_{\varphi_{*}, w}} \leq 4 C\|f\|_{\left(\Lambda_{\varphi, w}\right)^{\prime}} .
$$

Now suppose that $f \in M_{\varphi_{*}, w}$ and $\|f\|_{M_{\varphi_{*}, w}} \leq 1$. This implies that

$$
\int_{I} \varphi_{*}\left(\frac{f^{*}(t)}{w(t)}\right) w(t) d t \leq 1
$$


Taking $f_{0}=w$ and $f_{1}=\varphi_{*}\left(f^{*} / w\right) w$, we have $f_{0} \in M_{S}, f_{1} \in L^{1},\left\|f_{0}\right\|_{M_{S}}=1$ and $\left\|f_{1}\right\|_{L^{1}} \leq 1$. Since

$$
f^{*}(t)=\widehat{\psi}\left(f_{0}(t), f_{1}(t)\right)
$$

for all $t \in I$, we get $f^{*} \in \widehat{\psi}\left(M_{S}, L^{1}\right)$ and $\left\|f^{*}\right\|_{\left(\Lambda_{\varphi, w}\right)^{\prime}}=\left\|f^{*}\right\|_{\widehat{\psi}}^{1} \leq 2$. In consequence $f^{*} \in\left(\Lambda_{\varphi, w}\right)^{\prime}$, and since the Köthe dual of a symmetric space is also a symmetric space (see [9]), we get $f \in\left(\Lambda_{\varphi, w}\right)^{\prime}$. Combining the above inequalities, we obtain

$$
2^{-1}\|f\|_{\left(\Lambda_{\varphi, w}\right)^{\prime}} \leq\|f\|_{M_{\varphi_{*}, w}} \leq 4 C\|f\|_{\left(\Lambda_{\varphi, w}\right)^{\prime}}
$$

for every $f \in\left(\Lambda_{\varphi, w}\right)^{\prime}$, where $C$ is the regularity constant of $w$.

(ii). Fix $x \in I$ and let $f=\chi_{(0, x)} / x$. Since $\Lambda_{\varphi, w}$ has the Fatou property, it follows by Theorem $1 \mathrm{~b}$ ) that there exist functions $g \in E:=\Lambda_{\varphi, w}, h \in E^{\prime}$ such that $f=g h$ and $\|g\|_{E}=\|h\|_{E^{\prime}}=1$. Thus, we have $\|f / h\|_{E}=\|h\|_{E^{\prime}}=1$. Without loss of generality we can assume that $h=h^{*}$ and $\operatorname{supp} h=(0, x)$ (cf. [6, pp. 38-41]). In consequence

$$
\begin{aligned}
\int_{0}^{x} \varphi\left(\frac{1}{x h(t)}\right) w(x-t) d t & =\int_{0}^{x} \varphi\left(\frac{1}{x h(x-t)}\right) w(t) d t \\
& =\int_{I} \varphi\left(\left(\frac{\chi_{(0, x)}}{x h}\right)^{*}(t)\right) w(t) d t \leq 1
\end{aligned}
$$

Moreover, since $\|h\|_{E^{\prime}}=1$, we have $\|h\|_{M_{\varphi_{*}, w}} \leq K$ by Lemma 2 , and so

$$
\int_{I} \varphi_{*}\left(\frac{h(t)}{K w(t)}\right) w(t) d t \leq 1
$$

From $\varphi \in \Delta_{2}$, there exists $C \geq 2$ such that $\varphi(2 t) \leq C \varphi(t)$ for all $t>0$. This implies that $\varphi(\lambda t) \leq 2^{p} \lambda^{p} \varphi(t)$ for $p=\ln C / \ln 2 \geq 1$ and all $\lambda \geq 1, t \geq 0$. Since $w$ is nonincreasing, we also have

$$
\frac{w(x-t)}{w(t)} \geq 1 \text { for any } t \in[x / 2, x]
$$

Combining the above inequalities with the definition of the conjugate function $\varphi_{*}$, we obtain

$$
\begin{aligned}
\frac{1}{x K} \int_{x / 2}^{x}\left(\frac{w(x-t)}{w(t)}\right)^{1 / p} d t & \leq \int_{x / 2}^{x} \varphi_{*}\left(\frac{h(t)}{K w(t)}\right) w(t) d t \\
& +\int_{x / 2}^{x} \varphi\left(\frac{1}{x h(t)}\left(\frac{w(x-t)}{w(t)}\right)^{1 / p}\right) w(t) d t \\
& \leq 1+2^{p} \int_{x / 2}^{x} \varphi\left(\frac{1}{x h(t)}\right) w(x-t) d t \leq 1+2^{p}
\end{aligned}
$$

Clearly

$$
\frac{1}{x} \int_{0}^{x / 2}\left(\frac{w(x-t)}{w(t)}\right)^{1 / p} d t \leq \frac{1}{2}
$$

Hence for $K_{1}=\left(1+2^{p}\right) K+1$,

$$
\sup _{x \in I} \frac{1}{x} \int_{0}^{x}\left(\frac{w(x-t)}{w(t)}\right)^{1 / p} d t \leq K_{1} .
$$

This is equivalent to regularity of $w^{1 / p}$.

In the case when $\varphi(t)=t, p=1$ and obviously $w$ is regular. 
Now we assume that $\varphi \in \Delta_{2}$ is an $N$-function and we shall show that $w$ is regular. It is easy to check that the regularity of $w^{a}$ for some $0<a<\infty$ implies (cf. [12], Lemma 3)

$$
w(t) \asymp w(2 t) \text { for all } t \in I \text { with } 2 t \in I .
$$

Now let $h \in M_{S}$ with $\|h\|_{M_{S}} \leq 1$. For any $f \in L^{1}$ with $\|f\|_{L^{1}} \leq 1$, we have

$$
\widehat{\psi}(|h|,|f|) \in\left(\Lambda_{\varphi, w}\right)^{\prime}=\widehat{\psi}\left(M_{S}, L^{1}\right)
$$

with $\|\widehat{\psi}(|h|,|f|)\|_{\left(\Lambda_{\varphi, w}\right)^{\prime}} \leq 2$. Now if $\left(\Lambda_{\varphi, w}\right)^{\prime}=M_{\varphi_{*}, w}$, it follows by Lemma 2 that there is $K>0$ so that

$$
\sup _{\|f\|_{L^{1}} \leq 1}\|\widehat{\psi}(|h|,|f|)\|_{M_{\varphi_{*}, w}} \leq 2 K .
$$

Taking $f=(1 / x) \chi_{(0, x)}$ with $x \in I$, we get by the homogeneity of $\widehat{\psi}$

$$
\int_{0}^{x} \varphi_{*}\left(\frac{\widehat{\psi}\left(x h^{*}(t), 1\right)}{2 K x w(t)}\right) w(t) d t \leq \int_{I} \varphi_{*}\left(\frac{\widehat{\psi}(|h|,|f|)^{*}(t)}{2 K w(t)}\right) w(t) d t \leq 1 .
$$

Combining these with $w(t) \leq c w(2 t)$ (by $w(t) \asymp w(2 t))$ gives that for all $x \in I$,

$$
\frac{x}{2} \varphi_{*}\left(\frac{\widehat{\psi}\left(x h^{*}(x), 1\right)}{2 c K x w(x)}\right) w(x) \leq \int_{x / 2}^{x} \varphi_{*}\left(\frac{\widehat{\psi}\left(x h^{*}(t), 1\right)}{2 K x w(t)}\right) w(t) d t \leq 1 .
$$

Hence for all $x \in I$,

$$
\begin{aligned}
x h^{*}(x) \varphi_{*}^{-1}\left(\frac{1}{x h^{*}(x)}\right) & =\widehat{\psi}\left(x h^{*}(x), 1\right) \\
& \leq 2 c K x w(x) \varphi_{*}^{-1}\left(\frac{2}{x w(x)}\right) .
\end{aligned}
$$

Now since for any $N$-function it holds $u \leq \varphi^{-1}(u) \varphi_{*}^{-1}(u) \leq 2 u$ for all $u \geq 0$, we get

$$
\varphi^{-1}\left(\frac{2}{x w(x)}\right) \leq 8 c K \varphi^{-1}\left(\frac{1}{x h^{*}(x)}\right) \text { for all } x \in I,
$$

which in view of the $\Delta_{2}$-condition implies that

$$
\varphi^{-1}\left(\frac{2}{x w(x)}\right) \leq \varphi^{-1}\left(\frac{C}{x h^{*}(x)}\right)
$$

for all $x \in I$ and a constant $C>0$, and finally it yields that

$$
\sup _{x \in I} \frac{h^{*}(x)}{w(x)}<\infty \text {. }
$$

In consequence we proved that

$$
\left(\Lambda_{w}\right)^{\prime}=M_{S}=\left\{h \in L^{0}(m): \frac{h^{*}}{w} \in L^{\infty}\right\} .
$$

Thus, by the above proof for $\varphi(t)=t$, it follows that $w$ is regular.

If the Orlicz function $\varphi$ is neither linear nor an $N$-function, we have the following description of the Köthe dual spaces of Orlicz-Lorentz spaces.

Theorem 3. Let $\varphi$ be an Orlicz function and let $w$ be a regular weight. Then the following holds true:

(i) If $\lim _{t \rightarrow 0} \varphi(t) / t>0$ and $\lim _{t \rightarrow 0} \varphi(t) / t<\infty$, then $\varphi(t) \asymp t$ and

$$
\left(\Lambda_{\varphi, w}\right)^{\prime}=M_{S} .
$$


(ii) If $\lim _{t \rightarrow 0} \varphi(t) / t>0$ and $\lim _{t \rightarrow \infty} \varphi(t) / t=\infty$, then there exists an $N$-function $\phi$ such that $\phi(t) \asymp t^{2}$ for $t$ small enough and $\phi(t) \asymp \varphi(t)$ for $t$ large enough, and

$$
\left(\Lambda_{\varphi, w}\right)^{\prime}=M_{S}+M_{\phi_{*}, w} .
$$

(iii) If $\lim _{t \rightarrow 0} \varphi(t) / t=0$ and $\lim _{t \rightarrow \infty} \varphi(t) / t<\infty$, then there exists an $N$-function $\phi$ such that $\phi(t) \asymp \varphi(t)$ for $t$ small enough, and $\phi(t) \asymp t$ for $t$ large enough, and

$$
\left(\Lambda_{\varphi, w}\right)^{\prime}=M_{S} \cap M_{\phi_{*}, w} .
$$

Proof. We will use two particular cases of the Lozanovskiu duality result from which it follows that for any couple $\left(E_{0}, E_{1}\right)$ of Banach function spaces defined on the same measure space, we have

$$
\left(E_{0} \cap E_{1}\right)^{\prime}=E_{0}^{\prime}+E_{1}^{\prime} \text { and }\left(E_{0}+E_{1}\right)^{\prime}=E_{0}^{\prime} \cap E_{1}^{\prime},
$$

where $E_{0} \cap E_{1}$ and $E_{0}+E_{1}$ are equipped with the natural interpolation norms (cf. [3] or [9]).

(i). It is obvious since in this case $\Lambda_{\varphi, w}=\Lambda_{w}$.

(ii). We may assume without loss of generality that $\varphi(1)=1$. Then it is easy to check that

$$
\varphi(t) \asymp \max \{t, \phi(t)\},
$$

where $\phi(t)=t^{2}$ for $t \leq 1$ and $\phi(t)=2 \varphi(t)-1$ for $t>1$. Clearly

$$
\Lambda_{\varphi, w}=\Lambda_{w} \cap \Lambda_{\phi, w} .
$$

Thus the required result follows by Theorem 2 and the Lozanovskiı result mentioned above.

(iii). We define $\varphi_{1}(t):=\varphi(t)$ if $t \leq 1$ and $\varphi_{1}(t):=\varphi_{+}^{\prime}(1) t^{2}+\left(1-\varphi_{+}^{\prime}(1)\right)$ for $t>1$ and

$$
\phi(t):=\int_{0}^{t} \min \left\{s, \varphi_{1}(s)\right\} \frac{d s}{s} .
$$

Again it is easy to check that $\phi$ satisfies the required conditions. Since $\varphi(t) \asymp$ $\min \{t, \phi(t)\}$, we immediately obtain

$$
\Lambda_{\varphi, w}=\Lambda_{w}+\Lambda_{\phi, w} .
$$

Thus again Theorem 2 and the Lozanovskiı̌ result apply.

By [7] (cf. also [5]), we have that $\Lambda_{\varphi, w}$ is separable if and only if $\varphi$ satisfies the corresponding $\Delta_{2}$-condition. Thus if the weight function $w$ is regular, then by Theorems 2 and 3 we obtain a description of the Banach dual space of the Orlicz-Lorentz space. In fact the following corollary of Theorem 2 holds true.

Theorem 4. Let either $\varphi(t)=t$ or $\varphi$ be an $N$-function satisfying the $\Delta_{2}$-condition. Then the regularity of the weight function $w$ is a necessary and sufficient condition for the coincidence of the Banach dual space of the Orlicz-Lorentz space $\Lambda_{\varphi, w}$ with the space $M_{\varphi_{*}, w}$.

Analogous results characterizing the Köthe dual or dual spaces may also be stated for Orlicz-Lorentz sequence spaces.

Finally we notice that the description of the dual of the Lorentz space $\Lambda_{p, w}$ given by Reisner [12] is a particular case of the above theorem for $\varphi(t)=t^{p}$ for $t \geq 0$, $1 \leq p<\infty$. 


\section{REFERENCES}

[1] G.D. Allen, Duals of Lorentz spaces, Pacific J. Math. 77 (1978), 287-291. MR 80b:46015

[2] G.D. Allen and L.C. Shen, On the structure of principal ideals of operators, Trans. Amer. Math. Soc. 238 (1978), 253-270. MR 57:7230

[3] C. Bennett and R. Sharpley, Interpolation of Operators, Pure and Applied Mathematics 129, Academic Press, Boston 1988. MR 89e:46001

[4] A.P. Calderón, Intermediate spaces and interpolation, the complex method, Studia Math. 24 (1964), 113-190. MR 29:5097

[5] H. Hudzik, A. Kamińska and M. Mastyło, Geometric properties of some Calderón-Lozanovskiı spaces and Orlicz-Lorentz spaces, Houston J. Math. 22 (1996), 639-663. MR 97i:46057

[6] N.J. Kalton, Nonlinear commutators in interpolation theory, Memoirs Amer. Math. Soc., Providence, RI 373 (1988). MR 89h:47104

[7] A. Kamińska, Some remarks on Orlicz-Lorentz spaces, Math. Nachr. 147 (1990), 29-38. MR 92h:46034

[8] L.V. Kantorovich and G.P. Akilov, Functional Analysis, 2nd rev. ed., "Nauka", Moscow 1977 (in Russian); English transl.: Pergamon Press 1982. MR 58:23465; MR 83h:46002

[9] S.G. Krein, Ju.I. Petunin and E.M. Semenov, Interpolation of Linear Operators, Amer. Math. Soc., Trans. of Math. Monog. 54, Providence, RI, 1982. MR 84j:46103

[10] G.Ya. Lozanovskiı̌, On some Banach lattices IV, Sibirsk. Math. Zh. 14 (1973), 140-155; English transl.: Sibirsk. Math. J. 14 (1973), 97-108. MR 49:1089

[11] G.Ya. Lozanovskiı̆, Transformations of ideal Banach spaces by means of concave functions, Qualitative and approximate methods for the investigation of operator equations, Gos. Univ., Yaroslavl. 3 (1978), 122-148 (in Russian). MR 83a:46042a

[12] S. Reisner, On the duals of Lorentz function and sequence spaces, Indiana Univ. Math. J. 31 (1982), 65-72. MR 83m:46044

[13] S. Reisner, On two theorems of Lozanovski乞 concerning intermediate Banach lattices, Lecture Notes in Math. 1317 (1988), 67-83. MR 89j:46019

Faculty of Mathematics and Computer Science, A. Mickiewicz University, Matejki 48/49, 60-769 Poznań, Poland and Institute of Mathematics, Poznań University of TechNOLOGy, Piotrowo 3A, 60-965 Poznań, Poland

E-mail address: hudzik@amu.edu.pl

Department of Mathematical Sciences, The University of Memphis, Memphis, TenNESSEE 38152

E-mail address: kaminska@memphis.edu

Faculty of Mathematics and Computer Science, A. Mickiewicz University, Matejki 48/49, 60-769 Poznań, Poland

E-mail address: mastylo@amu.edu.pl 